\title{
Distribution of inorganics and trace elements during waste gasification in a bench-scale fluidized-bed
}

Authors: Vanessa Ferreira de Almeida ${ }^{1}$, Alberto Gómez-Barea ${ }^{1,3^{*}}$, Susanna Louise Nilsson ${ }^{1}$, Sanna Tuomi ${ }^{2}$

${ }^{1}$ Departamento de Ingeniería Química y Ambiental, Universidad de Sevilla, Camino de los Descubrimientos s/n, 41092 Seville, Spain

${ }^{2}$ VTT Technical Research Centre of Finland, P.O. Box 1000, FI-02044 VTT, Finland

${ }^{3}$ Laboratory of Engineering for Energy and Environmental Sustainability, Universidad de Sevilla, Camino de los Descubrimientos s/n, 41092 Seville, Spain

*agomezbarea@us.es

\section{Supporting information}

\section{S1: List of tars compounds measured in the tests}

Table S1 contains the 53 tar compounds that were quantified with GC:

Table S1: List if tar compounds quantified by GC
\begin{tabular}{|l|l|}
\hline$\#$ & Compound \\
\hline 1 & benzene \\
\hline 2 & pyridine \\
\hline 3 & 1 H-pyrrole \\
\hline 4 & toluene \\
\hline 5 & ethylbenzene \\
\hline 6 & m-xylene \\
\hline 7 & ethynylbenzene \\
\hline 8 & styrene \\
\hline 9 & o-xylene \\
\hline
\end{tabular}




\begin{tabular}{|c|c|}
\hline 10 & benzaldehyde \\
\hline 11 & phenol \\
\hline 12 & benzonitrile \\
\hline 13 & 4-methylstyrene \\
\hline 14 & indene \\
\hline 15 & o-cresol \\
\hline 16 & $m+p-c r e s o l$ \\
\hline 17 & naphthalene \\
\hline 18 & quinolone \\
\hline 19 & quinazoline \\
\hline 20 & isoquinoline \\
\hline 21 & 1H-indole \\
\hline 22 & 2-methylnaphthalene \\
\hline 23 & 1-methylnaphthalen \\
\hline 24 & biphenyl \\
\hline 25 & 1.6 dimethylnaphtalene \\
\hline 26 & 2-ethylnaphthalene \\
\hline 27 & acenaphtylene \\
\hline 28 & acenaphthene \\
\hline 29 & dibenzofuran \\
\hline 30 & bibenzyl \\
\hline 31 & 2-methyl-1-naphthol \\
\hline 32 & fluorine \\
\hline 33 & phenanthrene \\
\hline 34 & anthracene \\
\hline 35 & carbazole \\
\hline 36 & 1-phenylnaphthalene \\
\hline 37 & 2-methylanthracene \\
\hline 38 & 4H-cyclopenta(def)phenanthrene \\
\hline 39 & fluoranthene \\
\hline 40 & pyrene \\
\hline 41 & 2,3-benzofluoren \\
\hline 42 & 1,1 binaphthyl \\
\hline 43 & 1,2 benzanthracene \\
\hline 44 & chrysene \\
\hline 45 & 2,3 benzanthracene \\
\hline
\end{tabular}




\begin{tabular}{|l|l|}
\hline 46 & benzo(b)fluoranthene \\
\hline 47 & benzo(e)pyrene \\
\hline 48 & benzo(a)pyrene \\
\hline 49 & perylene \\
\hline 50 & indeno(1,2,3-cd) pyrene \\
\hline 51 & benzo(ghi)perylene \\
\hline 52 & anthanthrene \\
\hline 53 & coronene \\
\hline
\end{tabular}

\section{S2: Concentration of tars $\left(\mathrm{mg} / \mathrm{m}^{3}{ }_{\mathrm{n}}\right)$ measured in the tests and other tars related data}

The following Table S2 contains the concentrations of tars per test $(1-6)$ and other tars related data:

Table S2. Average of the concentrations of tars per test and other tars related data

\begin{tabular}{|c|c|c|c|c|c|c|}
\hline $\begin{array}{l}\text { CONCENTRATION (mg/m3n) / } \\
\text { TEST }\end{array}$ & 1 & 2 & 3 & 4 & 5 & 6 \\
\hline Benzene & 17919.5 & 23646.2 & 14331.0 & 14664.8 & 20343.9 & 16844.5 \\
\hline Pyridine* & 0.0 & 0.0 & 0.0 & 0.0 & 0.0 & 0.0 \\
\hline 1H-Pyrrole* & 0.0 & 0.0 & 0.0 & 0.0 & 0.0 & 0.0 \\
\hline Toluene & 890.0 & 1110.3 & 692.2 & 699.7 & 958.0 & 1129.9 \\
\hline Ethylbenzene & 0.0 & 0.0 & 0.0 & 0.0 & 0.0 & 0.0 \\
\hline m-Xylene & 0.0 & 0.0 & 0.0 & 0.0 & 0.0 & 0.0 \\
\hline Ethynylbenzene & 169.8 & 216.9 & 166.5 & 151.1 & 275.0 & 189.6 \\
\hline Styrene & 1608.7 & 1894.2 & 1248.3 & 1247.8 & 1882.2 & 1758.4 \\
\hline o-Xylene & 0.0 & 0.0 & 0.0 & 0.0 & 0.0 & 0.0 \\
\hline Benzaldehyde* & 0.0 & 0.0 & 0.0 & 0.0 & 0.0 & 0.0 \\
\hline Phenol & 0.0 & 0.0 & 0.0 & 0.0 & 0.0 & 0.0 \\
\hline Benzonitrile* & 93.1 & 120.1 & 86.3 & 77.4 & 124.9 & 49.6 \\
\hline 4-Methylstyrene & 42.2 & 48.2 & 41.7 & 39.9 & 51.5 & 33.3 \\
\hline Indene & 725.9 & 944.5 & 582.5 & 578.5 & 898.0 & 741.1 \\
\hline o-Cresol & 0.0 & 0.0 & 0.0 & 0.0 & 0.0 & 0.0 \\
\hline$m+p-C r e s o l$ & 0.0 & 0.0 & 0.0 & 0.0 & 0.0 & 0.0 \\
\hline Naphthalene & 5759.1 & 7837.9 & 4455.5 & 4565.5 & 6880.3 & 5151.4 \\
\hline
\end{tabular}




\begin{tabular}{|c|c|c|c|c|c|c|}
\hline Quinoline* $^{*}$ & 34.7 & 49.4 & 21.8 & 29.5 & 46.5 & 0.0 \\
\hline Quinazoline* & 0.0 & 0.0 & 0.0 & 0.0 & 0.0 & 0.0 \\
\hline Isoquinoline* & 0.0 & 0.0 & 0.0 & 0.0 & 0.0 & 0.0 \\
\hline $1 \mathrm{H}-$ Indole ${ }^{*}$ & 0.0 & 0.0 & 0.0 & 0.0 & 0.0 & 0.0 \\
\hline 2-Methylnaphthalene & 160.6 & 197.7 & 124.6 & 124.6 & 167.3 & 169.0 \\
\hline 1-Methylnaphthalene & 90.5 & 120.1 & 68.9 & 69.3 & 106.7 & 90.0 \\
\hline Biphenyl & 439.7 & 555.1 & 343.7 & 350.1 & 517.1 & 429.5 \\
\hline 2-Ethylnaphthalene & 0.0 & 0.0 & 0.0 & 0.0 & 0.0 & 0.0 \\
\hline 1.6 Dimethylnaphtalene & 255.9 & 320.0 & 193.6 & 192.8 & 320.7 & 251.7 \\
\hline Acenaphtylene & 1087.5 & 1502.1 & 884.8 & 902.3 & 1361.5 & 926.9 \\
\hline Acenaphthene & 79.6 & 100.3 & 49.2 & 38.6 & 64.5 & 61.9 \\
\hline Dibenzofuran & 54.9 & 60.0 & 60.9 & 58.7 & 58.6 & 0.0 \\
\hline Bibenzyl & 0.0 & 14.0 & 0.0 & 0.0 & 30.3 & 0.0 \\
\hline 2-Methyl-1-Naphthol* & 0.0 & 17.2 & 0.0 & 0.0 & 15.8 & 0.0 \\
\hline Fluorene & 315.5 & 425.0 & 242.9 & 243.4 & 396.8 & 300.9 \\
\hline Phenanthrene & 1285.9 & 1810.3 & 1025.0 & 989.2 & 1593.0 & 1085.9 \\
\hline Anthracene & 298.5 & 427.1 & 236.8 & 222.4 & 382.5 & 256.3 \\
\hline Carbazole* $^{*}$ & 0.0 & 0.0 & 0.0 & 0.0 & 0.0 & 0.0 \\
\hline 1-Phenylnaphthalene & 34.1 & 47.1 & 26.7 & 23.9 & 44.6 & 29.5 \\
\hline 2-Methylanthracene & 55.4 & 71.3 & 37.8 & 30.7 & 38.4 & 28.6 \\
\hline 4H-Cyclopenta(def)Phenanthrene & 95.4 & 136.9 & 76.1 & 71.5 & 124.8 & 78.2 \\
\hline Fluoranthene & 465.7 & 686.1 & 389.2 & 366.1 & 603.7 & 332.5 \\
\hline Pyrene & 537.5 & 784.0 & 422.5 & 397.7 & 704.7 & 406.1 \\
\hline 2,3-Benzofluorene & 45.2 & 62.2 & 36.1 & 34.3 & 60.0 & 41.6 \\
\hline 1,1 Binaphthyl ${ }^{*}$ & 0.0 & 0.0 & 0.0 & 0.0 & 0.0 & 0.0 \\
\hline 1,2 Benzanthracene & 136.1 & 195.3 & 116.4 & 109.2 & 199.5 & 119.8 \\
\hline Chrysene & 155.3 & 222.8 & 132.1 & 126.9 & 220.6 & 140.0 \\
\hline 2,3 Benzanthracene & 0.0 & 0.0 & 0.0 & 0.0 & 0.0 & 0.0 \\
\hline Benzo(b)fluoranthene & 73.7 & 104.7 & 67.6 & 65.4 & 108.0 & 64.8 \\
\hline Benzo(e)pyrene & 73.0 & 107.0 & 62.3 & 61.8 & 105.8 & 64.4 \\
\hline Benzo(a)pyrene & 152.5 & 220.5 & 131.2 & 131.9 & 220.5 & 129.0 \\
\hline Perylene & 0.0 & 0.0 & 0.0 & 0.0 & 0.0 & 0.0 \\
\hline Indeno(1,2,3-cd) pyrene & 48.5 & 63.8 & 39.0 & 40.3 & 73.8 & 39.4 \\
\hline Benzo(ghi)perylene & 42.5 & 61.6 & 34.3 & 38.7 & 63.9 & 34.5 \\
\hline Anthanthrene & 51.0 & 64.7 & 25.2 & 31.3 & 75.0 & 36.6 \\
\hline Coronene & 0.0 & 0.0 & 0.0 & 0.0 & 0.0 & 0.0 \\
\hline IDENTIFIED: & $\mathrm{mg} / \mathrm{m}^{3} \mathrm{n}$ & $\mathrm{mg} / \mathrm{m}^{3} \mathrm{n}$ & $\mathrm{mg} / \mathrm{m}^{3} \mathrm{n}$ & $\mathrm{mg} / \mathrm{m}^{3} \mathrm{n}$ & $\mathrm{mg} / \mathrm{m}^{3} \mathrm{n}$ & $\mathrm{mg} / \mathrm{m}^{3} \mathrm{n}$ \\
\hline IDENTIFIED TARS & 15358.1 & 20598.4 & 12121.8 & 12110.5 & 18774.5 & 14170.6 \\
\hline IDENTIFIED TARS+BENZENE & 33277.6 & 44244.6 & 26452.8 & 26775.4 & 39118.4 & 31015.1 \\
\hline UNIDENTIFIED INCLUDED: & $\mathrm{mg} / \mathrm{m}^{3} \mathrm{n}$ & $\mathrm{mg} / \mathrm{m}^{3} \mathrm{n}$ & $\mathrm{mg} / \mathrm{m}^{3} \mathrm{n}$ & $\mathrm{mg} / \mathrm{m}^{3} \mathrm{n}$ & $\mathrm{mg} / \mathrm{m}^{3} \mathrm{n}$ & $\mathrm{mg} / \mathrm{m}^{3} \mathrm{n}$ \\
\hline
\end{tabular}




\begin{tabular}{|l|c|c|c|c|c|c|} 
TARS < NAPHTHALENE & 3673.8 & 4608.2 & 2970.7 & 2821.4 & 4517.9 & 4120.4 \\
\hline NAPHTHALENE & 5759.1 & 7837.9 & 4455.5 & 4565.5 & 6880.3 & 5151.4 \\
\hline HEAVIER TARS & 8670.1 & 12436.7 & 6795.5 & 6642.1 & 11883.7 & 7293.3 \\
\hline Unidentified & 2744.9 & 4284.4 & 2099.9 & 1918.5 & 4507.4 & 2394.5 \\
\hline TOTAL (unidentified included) & 18103.0 & 24882.8 & 14221.7 & 14029.0 & 23281.9 & 16565.0 \\
\hline Unidentified (\%) & $7.6 \%$ & $8.8 \%$ & $7.4 \%$ & $6.7 \%$ & $10.3 \%$ & $7.2 \%$ \\
\hline Identified (\%) & $92.4 \%$ & $91.2 \%$ & $92.6 \%$ & $93.3 \%$ & $89.7 \%$ & $92.8 \%$ \\
\hline TOTAL (\%) & $100.0 \%$ & $100.0 \%$ & $100.0 \%$ & $100.0 \%$ & $100.0 \%$ & $100.0 \%$ \\
\hline $\mathrm{C}-\%$ & 92.9 & 92.9 & 92.9 & 92.9 & 92.9 & 92.9 \\
\hline $\mathrm{H}-\%$ & 7.1 & 7.1 & 7.1 & 7.2 & 7.1 & 7.2 \\
\hline $\mathrm{N}-\%$ & 0.0 & 0.0 & 0.1 & 0.1 & 0.1 & 0.0 \\
\hline $\mathrm{O}-\%$ & 0.0 & 0.0 & 0.0 & 0.0 & 0.0 & 0.0 \\
\hline $\mathrm{H}_{2} \mathrm{O}, \mathrm{g} / \mathrm{m}^{3} \mathrm{n}$ (per dry gas) & 75.1 & 179.2 & 75.0 & 70.4 & 79.7 & 66.2 \\
\hline
\end{tabular}

* compounds containing $N$ 